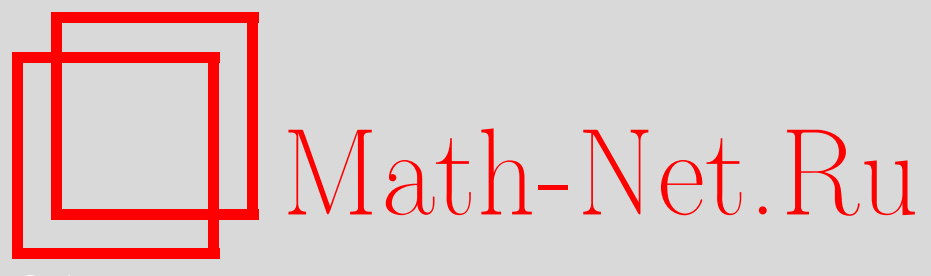

В. М. Бухштабер, О. В. Карпов, С. И. Тертычный, Система на торе, моделирующая динамику перехода Джозефсона, УМН, 2012, том 67, выпуск 1, 181-182

DOI: https://doi.org/10.4213/rm9456

Использование Общероссийского математического портала Math-Net.Ru подразумевает, что вы прочитали и согласны с пользовательским соглашением http://www.mathnet.ru/rus/agreement

Параметры загрузки:

IP : 54.197 .130 .99

26 апреля 2023 г., 15:43:10

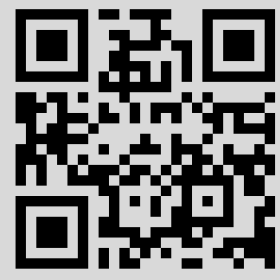




\section{Система на торе, моделирующая динамику перехода Джозефсона}

\section{В. М. Бухштабер, О. В. Карпов, С. И. Тертычный}

Работа посвящена развитию теории уравнения (см. [1], [2], а также [3])

$$
\dot{\varphi}(t)+\sin \varphi(t)=i_{0}+i_{1} \sin \mu t,
$$

где $i_{0}, i_{1}, \mu$ - константы. В первую очередь нас интересуют задачи, пришедшие из физики сильношунтированного перехода Джозефсона в сверхпроводниках, динамику которого успешно моделирует это уравнение (см. [4]-[7]).

Положив $x=\mu t+c$, где $c=$ const, и $y=\varphi(t)$, мы получаем динамическую систему на торе

$$
\dot{x}=\mu, \quad \dot{y}=i_{0}+i_{1} \sin x-\sin y .
$$

Отметим, что при $\mu i_{1} \neq 0$ система (2) эквивалентна уравнению (1). Пусть $x(t)=$ $\mu t+c, y(t)=y\left(t ; i_{0}, i_{1}, \mu\right)$ - решение этой системы. Классическое число вращения $\nu=$ $\lim _{t \rightarrow \infty} y(t) / x(t)$ (см. [8]) не зависит от начальных данных $(x(0), y(0))$ и задает функцию $\nu=\nu\left(i_{0}, i_{1}, \mu\right)$ в $\mathbb{R}^{3}$. Например, $\nu(\mu, 1, \mu) \equiv 1$.

Область $Q_{m}=\left\{\left(i_{0}, i_{1}, \mu\right) \in \mathbb{R}^{3}: \nu=m\right\}$, где $m=0, \pm 1, \ldots$, называется областью квантования порядка $m$ (см. [9]). Такое название объясняется следующим.

Положим $y_{n}(t)=y(t+n T)$, где $T=2 \pi / \mu$, и $q_{n}(t)=y_{n+1}(t)-y_{n}(t)$. В [9] доказано, что если последовательность функций $\left\{q_{n}(t), n=0,1, \ldots\right\}$ сходится при фиксированном $t$, то $\lim q_{n}(t)=2 \pi \nu$ и, в частности, предел не зависит от $t$. Более того, в этом случае $\nu$ обязано быть целым числом и имеет физическую интерпретацию - номер ступеньки Шапиро.

Задачи: (i) описать карты областей $Q_{m}$ в $\mathbb{R}^{3}$ и в плоскостях $\left\{\left(i_{0}, i_{1}\right)\right\},\left\{\left(i_{0}, \mu\right)\right\}$, $\left\{\left(i_{1}, \mu\right)\right\}$ при фиксированных значениях $\mu, i_{1}, i_{0}$ соответственно;

(ii) описать динамику карт областей $Q_{m}$ в указанных плоскостях при изменении фиксированных значений $\mu, i_{1}$ и $i_{0}$.

Пусть $\varphi_{k}(t)=\varphi_{k}\left(t ; i_{0}, i_{1}, \mu\right), k=1,2,-$ решения уравнения (1) с начальными данными $\varphi_{1}(0)=0$ и $\varphi_{2}(0)=\pi$ соответственно. Положим $\xi_{k}(t)=\int_{0}^{t} \cos \varphi_{k}(t) d t$. Используя теорему 1 работы [1], получаем, что точка $\left(i_{0}, i_{1}, \mu\right)$ лежит в области $Q_{m}$ для $m=\nu\left(i_{0}, i_{1}, \mu\right)$ тогда и только тогда, когда

$$
\begin{gathered}
\sin \left(\frac{1}{2}\left(\varphi_{2}(T)-\varphi_{1}(T)\right)\right)=\exp \left\{-\frac{1}{2}\left(\xi_{1}(T)+\xi_{2}(T)\right)\right\}, \\
\left|\exp \left\{\frac{1}{2} \xi_{1}(T)\right\} \cos \left(\frac{1}{2} \varphi_{1}(T)\right)+\exp \left\{\frac{1}{2} \xi_{2}(T)\right\} \sin \left(\frac{1}{2} \varphi_{2}(T)\right)\right| \geqslant 2 .
\end{gathered}
$$

В частности, если $\varphi_{1}\left(T ; i_{0}, i_{1}, \mu\right)=2 l \pi$ для целого $l$, то точка $\left(i_{0}, i_{1}, \mu\right)$ лежит в области $Q_{m}$ и на границе этой области, когда $\xi_{1}(T)=0$. Например, $\varphi_{1}(T ; \mu, 1, \mu)=2 \pi$ и $\xi_{1}(T)=0$, поэтому точка $(\mu, 1, \mu)$ лежит на границе области $Q_{1}$ для любого $\mu$.

Положим $\langle\sin \varphi\rangle=\lim _{n \rightarrow \infty} \frac{1}{T} \int_{n T}^{(n+1) T} \sin \varphi d t$. Из (1) следует, что если $\left(i_{0}, i_{1}, \mu\right) \in Q_{m}$, то $\langle\sin \varphi\rangle=i_{0}-m \mu$, в частности, $\left|i_{0}-m \mu\right| \leqslant 1$.

Непосредственная проверка показывает, что преобразование $x_{1}=\varepsilon_{1} x+\delta \pi, y_{1}=$ $\varepsilon_{2} y$, где $\varepsilon_{k}= \pm 1, k=1,2$, и $\delta=0$ или 1 , приводит к следующему преобразованию параметров системы $(2):\left(i_{0}, i_{1}, \mu\right) \mapsto\left(\varepsilon_{2} i_{0},(-1)^{\delta} \varepsilon_{1} \varepsilon_{2} i_{1}, \varepsilon_{1} \mu\right)$.

Таким образом, $\nu\left(\varepsilon_{2} i_{0},(-1)^{\delta} \varepsilon_{1} \varepsilon_{2} i_{1}, \varepsilon_{1} \mu\right)=\varepsilon_{1} \varepsilon_{2} \nu\left(i_{0}, i_{1}, \mu\right)$. Получаем

$$
\nu\left(-i_{0}, i_{1}, \mu\right)=-\nu\left(i_{0}, i_{1}, \mu\right), \quad \nu\left(i_{0},-i_{1}, \mu\right)=\nu\left(i_{0}, i_{1}, \mu\right), \quad \nu\left(i_{0}, i_{1},-\mu\right)=-\nu\left(i_{0}, i_{1}, \mu\right) .
$$

Поэтому достаточно изучить функцию $\nu\left(i_{0}, i_{1}, \mu\right)$ только при $i_{0} \geqslant 0, i_{1} \geqslant 0, \mu \geqslant 0$. 
Лемма 1. В случае системы (2) для любого $i \geqslant 0$ имеем:

$$
\begin{gathered}
\left(i_{0}-i\right)+\left(i_{1}-i\right) \sin x-\sin y \leqslant \dot{y} \leqslant\left(i_{0}+i\right)+\left(i_{1}-i\right) \sin x-\sin y, \\
\left(i_{0}-1\right)+i_{1} \sin x \leqslant \dot{y} \leqslant\left(i_{0}+1\right)+i_{1} \sin x .
\end{gathered}
$$

Используя теорему сравнения Чаплыгина (см. [10]), получаем

СледСтвиЕ 1. Пусть $\mu>0$, тогда для любого $i \geqslant 0$

$$
\nu\left(i_{0}-i_{1},\left|i_{1}-i\right|, \mu\right) \leqslant \nu\left(i_{0}, i_{1}, \mu\right) \leqslant \nu\left(i_{0}+i_{1},\left|i_{1}-i\right|, \mu\right) .
$$

Лемма 2. Пусть $i_{0} \geqslant 0, \mu>0 u i_{1}=0$, тогда $\mu \nu=\gamma\left(i_{0}\right)$, где $\gamma\left(i_{0}\right)=0$, если $i_{0} \leqslant 1, u \gamma\left(i_{0}\right)=\sqrt{i_{0}^{2}-1}$, если $i_{0} \geqslant 1$.

Используя лемму 2 , получаем, что имеет место

Лемма 3. Пусть $\mu>0$, тогда $\gamma\left(i_{0}-i_{1}\right) \leqslant \mu \nu\left(i_{0}, i_{1}, \mu\right) \leqslant \gamma\left(i_{0}+i_{1}\right)$, в частности, $\nu\left(i_{0}, i_{1}, \mu\right) \rightarrow 0$ nрu $\mu \rightarrow \infty u \nu\left(i_{0}, i_{1}, \mu\right) \rightarrow \infty$ npu $\left|i_{0}-i_{1}\right|>1 u \mu \rightarrow 0$.

СледСтвие 2. Пусть $\left|i_{0}-i_{1}\right| \leqslant 1 u\left|i_{0}+i_{1}\right| \leqslant 1$, тогда $\left(i_{0}, i_{1}, \mu\right) \in Q_{0}$ для любого $\mu$.

Следствие 3 . Пересечение области $Q_{m}, m \neq 0$, с областью $\left\{i_{0} \geqslant 1+i_{1}, i_{1} \geqslant 0\right\}$ содержится в области $\left\{\left|i_{0}-\sqrt{1+\mu^{2} m^{2}}\right| \leqslant i_{1}\right\}$, в частности, пересечением этой области с плоскостью $\left\{i_{1}=0\right\}$ является кривая $\left\{\left(i_{0}, \mu\right): i_{0}^{2}=1+m^{2} \mu^{2}\right\}$.

Интегрируя неравенства (5), получаем следующее утверждение.

Лемма 4. Пусть $\mu>0$, тогда $\left(i_{0}-1\right) \leqslant \mu \nu\left(i_{0}, i_{1}, \mu\right) \leqslant\left(i_{0}+1\right)$ для любого $i_{1}$.

Таким образом, имеет место

Tеорема 1. Пусть $\mu>0$, тогда

$$
\max \left\{i_{0}-1, \gamma\left(i_{0}-i_{1}\right)\right\} \leqslant \mu \nu\left(i_{0}, i_{1}, \mu\right) \leqslant \min \left\{i_{0}+1, \gamma\left(i_{0}+i_{1}\right)\right\} .
$$

СледСтвие 4. Пусть $\left(i_{0}, i_{1}, \mu\right) \in Q_{m} u \mu>0$, тогда

$$
\max \left\{i_{0}-1, \gamma\left(i_{0}-i_{1}\right)\right\} \leqslant \mu m \leqslant \min \left\{i_{0}+1, \gamma\left(i_{0}+i_{1}\right)\right\} .
$$

\section{Список литературы}

[1] В. М. Бухштабер, О. В. Карпов, С. И. Тертычный, УМН, 59:2(356) (2004), 187-188. [2] В. М. Бухштабер, О. В. Карпов, С. И. Тертычный, УМН, 63:3(381) (2008), 155-156. [3] Ю. С. Ильяшенко, Д. А. Рыжов, Д. А. Филимонов, Функи. анализ и его прил., 45:3 (2011), 41-54. [4] К. К. Лихарев, Б. Т. Ульрих, Системъ с джозефсоновскими контактами, Изд-во Моск. ун-та, М., 1978. [5] А. Бароне, Дж. Патерно, Эффект Джозефсона. Физика и применения, Мир, М., 1984. [6] В.М.Бухштабер, О.В.Карпов, С. И. Тертычный, ЖЭЭТФ, 124:3(9) (2003), 691-699. [7] O. V. Karpov, V. M. Buchstaber, S. I. Tertychniy, J. Niemeyer, O. Kieler, J. Appl. Phys., 104:9 (2008), 093910; Virtual Journal of Applications of Superconductivity, 15:10 (2008). [8] В.И. Арнольд, Геометрические методы в теории обыкновенных дифберенциалъных уравнений, МЦНМО, М., 2002. [9] В. М. Бухштабер, О.В.Карпов, С. И. Тертычный, $T M \Phi, ~ 162: 2 ~(2010), ~ 254-265 .[\mathbf{1 0 ]}$ Н. Н. Лузин, УМН, 6:6(46) (1951), 3-27.

B. M. Бухштабер (V. M. Buchstaber)

Математический институт им. В. А. Стеклова РАН

E-mail: buchstab@mi.ras.ru

O. В. Карпов (O. V. Karpov)

ВНИИФТРИ

E-mail: mera@vniiftri.org

\section{C. И. Тертычный (S. I. Tertychnyi)}

ВНИИФТРИ

E-mail: mera@vniiftri.org
Представлено С. П. Новиковым

Принято редколлегией

11.01.2012 